\title{
Subdural fat effusion complicating parenteral nutrition
}

\author{
A Rushforth, M A Green, M I Levene, J W L Puntis
}

Department of Paediatrics and

Child Health,

University of Leeds

A Rushforth

M I Levene

J W L Puntis

Department of

Forensic Pathology,

University of Sheffield

M A Green

Correspondence to:

Dr J W L Puntis,

Department of Paediatrics and

Child Health,

Level D,

Level D, Wing,

The General Infirmary

at Leeds,

at Leeds,

Leeds LS2 9NS.

Accepted 25 July 1991

(Arch Dis Child 1991;66:1350-1)

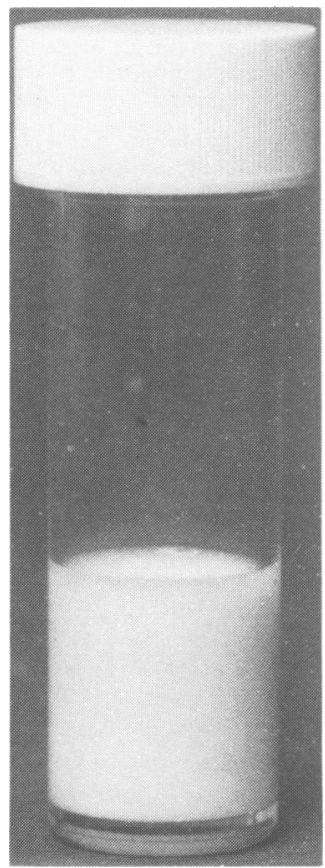

Figure 2 Milky white fluid aspirated by subdural tap with the biochemical composition of parenteral feeding fluids.

\begin{abstract}
A preterm infant fed parenterally through a central venous catheter developed a subdural effusion containing fat emulsion. Subsequent postmortem examination failed to demonstrate any vascular abnormality that might have explained this rarely reported complication. Although retrograde flow of feeding solutions into cerebral veins seems a likely explanation, the exact mechanism remains uncertain.
\end{abstract}

Technical problems may complicate parenteral nutrition. We report an unusual complication of a malpositioned central venous catheter, the aetiology of which remained obscure even after a full postmortem examination.

\section{Case report}

After the onset of premature labour at 26 weeks' gestation, a $925 \mathrm{~g}$ girl was delivered vaginally. She had an Apgar score of 6 at 1 minute and 9 at 5 minutes. Small bowel was noted to be prolapsing from a wound in the right iliac fossa that was caused by a penetrating abdominal injury to the mother two weeks earlier. The baby was ventilated from birth because of respiratory distress and transferred to the regional neonatal surgical unit. At laparotomy a small perforation of the ileum was resected and an end to end anastomosis performed. After surgery a percutaneous central venous catheter was advanced from an antecubital vein to the right atrium. The catheter was removed when full enteral feeding was achieved at the age of 13 days.

A patent ductus arteriosus and heart failure prevented weaning from the ventilator until surgical ligation at 16 days of age. Although respiratory function improved postoperatively, mechanical ventilation was again necessary after 48 hours because of a combination of infection and aspiration. Parenteral nutrition via a peripheral vein was reinstituted and continued over the next four weeks. During this time surgery was necessary to repair a left diaphragmatic hernia and postoperative recovery was complicated by peritonitis leading to a further laparotomy.

Gastro-oesophageal reflux and recurrent aspiration led to deterioration in respiratory function so that once again total parenteral nutrition was commenced. At 7 weeks of age a Vygon neonatal central venous feeding catheter was inserted percutaneously into the left posterior auricular vein. The catheter could be advanced no more than $9 \mathrm{~cm}$ and radiography indicated that the tip was probably in the external jugular vein. However, blood could be aspirated back with ease and so the catheter was used for parenteral feeding over the next 33 days. At this time the child became drowsy, suffered a convulsion, and was noted to have a full fontanelle. Ultrasound examination showed fluid in the subdural space (fig 1), into which a $23 \mathrm{G}$ butterfly needle was introduced; $24 \mathrm{ml}$ of milky white fluid were aspirated (fig 2).

\section{CLINICAL PROGRESS}

After aspiration of the subdural fluid the patient's neurological state returned to normal and the venous catheter was removed without any difficulty. Ultrasound examination showed no evidence of superior vena cava thrombosis and serial scans of the brain showed no reaccumulation of fluid or increase in ventricular size. Five weeks later death occurred from respiratory failure and a postmortem examination was performed.

\section{INVESTIGATIONS}

Microscopy of the aspirated subdural fluid showed no white or red blood cells and culture was sterile. Biochemical analysis revealed a glucose concentration of $44 \mu \mathrm{mol} / \mathrm{l}$ and triglyceride content of $22.3 \mu \mathrm{mol} / \mathrm{l}$ confirming that the fluid was largely composed of parenteral feeding solution and fat emulsion.

The postmortem examination demonstrated severe bronchopulmonary dysplasia. A detailed examination of the external jugular veins, sub-

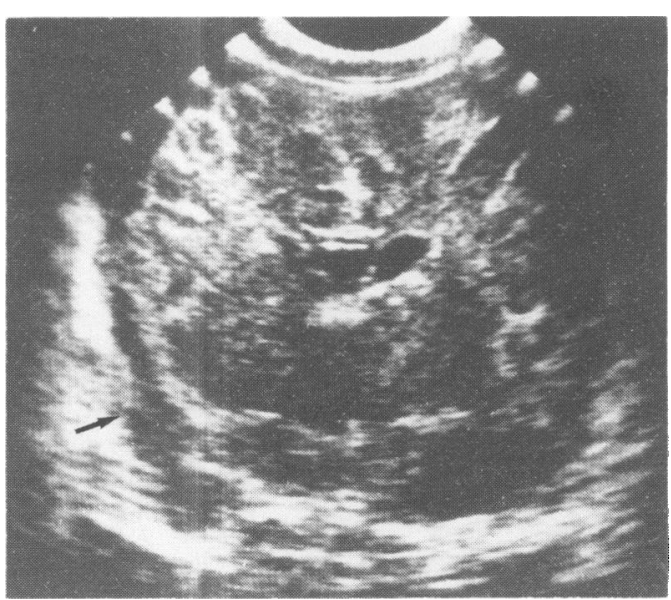

Figure 1 Coronal ultrasound scan of the brain showing a fluid filled space (arrow) between the brain and the skull from which lipid emulsion was aspirated. 
clavian veins, superior vena cava, and intracerebral venous sinuses showed no evidence of thrombosis or anatomical abnormality. Cystic changes were evident in both occipital poles of the brain.

\section{Discussion}

Percutaneous central venous catheters provide reliable venous access in the newborn ${ }^{1}$ and are widely used for delivering parenteral nutrition. ${ }^{2}$ In contrast to the major complication of sepsis, serious mechanical catheter related problems are rarely encountered. ${ }^{34}$ Two previous cases of parenteral feeding solution accumulating in the subdural space have been described in preterm infants but neither case came to postmortem examination. ${ }^{56}$ In one of these patients the catheter tip was situated in the superior vena cava and swelling of the neck several hours after onset of neurological symptoms suggested a relationship between subdural effusion and superior vena cava thrombosis. ${ }^{5}$ In the second case report the catheter tip was left in the temporal vein with no clinical evidence of vessel occlusion, although the authors postulated a probable septic thrombosis. ${ }^{6}$

In the only case that has been subjected to a full postmortem examination our findings indicate that a subdural collection of intravenous feeding fluid may occur in the absence of venous thrombosis or apparent anatomical abnormality. This being the case, we would agree with suggestions that retrograde flow of parenteral nutrition infusion along the internal jugular vein to the transverse sinus, sagittal sinus and, via a ruptured bridging vein into the subdural space, is the likely mechanism. Together with bronchopulmonary dysplasia, raised pulmonary vascular resistance might have been the cause of increased venous pressure giving rise to retrograde flow.

Subdural accumulation of intravenous feeding fluid appears to be a very rare complication of parenteral nutrition given via a misplaced central venous catheter. It would seem prudent whenever possible to site the tip of the catheter within the mid right atrium in an attempt to minimise the risk of venous thrombosis or retrograde flow. Acute onset of neurological symptoms in an infant with a central venous catheter should raise the possibility of a subdural effusion.

We would like to thank Dr S Variand for his help with the postmortem examination of this case.

1 Shaw JCL. Parenteral nutrition in the management of sick, low birthweight infants. Pediatr Clin North Am 1973;20: 333-58.

2 Puntis JWL. Percutaneous insertion of Silastic central venous feeding catheters. Intensive Therapy and Clinical Monitoring 1987;8:7-10.

3 Gladman G, Sinha S, Sims DG, Chiswick ML. Staphylococcus epidermidis and retention of neonatal percutaneous
central venous catheters. Arch Dis Child 1990;65:234-5. 4 Rubin S, Hewson P, Roberton NRC. Pulmonary complications of total parenteral nutrition in a neonate. $\mathcal{F} R$ Soc Med

5 Stine MJ, Harris $H$. Subdural collection of intravenous fat emulsion in a neonate. Clin Pediatr (Phila) 1985;24:40-1. 6 Young S, MacMahon P, Kovar IZ. Subdural intravenous fat collection: an unusual complication of central intravenous feeding in the neonate. Fournal of Parenteral and Enteral Nutrition 1989;13:661-2.

\title{
A national survey of nebuliser use
}

\author{
Helena J Childs, Carol A Dezateux
} Epidemiology, Institute of Child Health, 30 Guilford Street, London WCIN 1EH Helena J Childs Carol A Dezateux Correspondence to: Ms Childs.

Accepted 2 August 1991
Department of Paediatric

\begin{abstract}
Nebuliser drug delivery units were reused in $15 \%$ of paediatric wards participating in a national survey, while routine servicing and written information was provided by only half the wards issuing home nebulisers. Written information should be developed as a national resource, and further research on optimal cleaning practices is required.
\end{abstract}

Nebulisers are commonly used to treat children with severe asthma or cystic fibrosis in hospital and are increasingly prescribed for home treatment of very young children and those with chronic disease. While this may reduce the need for hospital admission, particularly of children with chronic conditions, it has been suggested (Arch Dis Child 1991;66:1351-3) that over-reliance on nebulised bronchodilators and inadequate supervision and education may increase the risk of life threatening asthmatic episodes. ${ }^{1}$ Nebuliser drug delivery units are manufactured for use by a single patient only, and though reuse may save money, potential hazards include bacterial contamination ${ }^{2}$ and loss of efficiency of drug delivery. A recent King's Fund conference concluded that reuse of items manufactured for single patient use was undesirable and recommended development of guidelines governing reuse at district levels. ${ }^{3}$

This survey aimed to determine current cleaning and reuse practices and to obtain information on the advice and support given to parents issued with a home nebuliser in a nationally representative sample of paediatric wards in England. We also sought to establish whether guidelines governing reuse were available at district health authority level. 